\title{
Expressed emotion among schizophrenic patients in Lagos, Nigeria: a pilot study
}

Follow-up treatment for patients with schizophrenia is to prevent relapse of their illness using not only pharmacological methods but also non-pharmacological approaches targeting such issues as expressed emotion. Expressed emotion (EE) can be described as an attitude displayed by family members or people in direct relationships with a patient that may have harmful effects and predict relapse. Of the five attributes of expressed emotion, critical comments, hostility and emotional over involvement have been shown to be most predictive of relapse. ${ }^{1}$ Expressed emotion can be rated as 'high' or 'low'.

Most studies support the hypothesis that high expressed emotion predicts relapse of schizophrenia. ${ }^{2}$ Brown and colleagues studied relapse rates of people in differing living arrangements after an episode of mental disorder, and found that relapse rates were 17\% for patients living by themselves or with siblings, $32 \%$ for those living with parents and 50\% for those living with spouses. ${ }^{3}$ Vaughn and Leff studied 43 patients with schizophrenia and 32 patients with depressive neurosis, and were able to follow up 37 and 30 patients respectively in the two groups. The relapse rate for the patients from homes that showed high expressed emotions was 50\%, compared with $12 \%$ for those from homes with low expressed emotion. ${ }^{4}$

Most studies on expressed emotion have used ratings derived from assessing or interviewing relatives, however expressed emotion can also be studied from the patient's perspective. ${ }^{5}$ Reicher and colleagues studied family members of 19 Nigerian schizophrenics using the Camberwell Family Interview and found that the proportion of high expressed emotion families increased progressively with the number of previous hospitalizations. ${ }^{6,7}$

Studies on expressed emotion amongst Africans deserve attention, especially so when one considers the findings of the International Pilot study of Schizophrenia which found that outcome was more favourable in the developing countries involved in the study, of which Nigeria was one. ${ }^{8}$ Whether difference in the trend of expressed emotion is partly responsible for this more favourable outcome can only be deduced through more research in this area.

The current study investigated expressed emotion among fifty schizophrenic patients and their relatives with the objective of determining how EE relates to socio demographic attributes of the subjects and the clinical course of the illness. The sample population was drawn from patients attending the Lagos University Teaching Hospital and the Neuropsychiatric

Correspondence

Dr RE Ogbolu

email:- emexxy@yahoo.com
Hospital, Yaba, Lagos, Nigeria. The consent of the institutions was sought and ethical approval granted from the health research and ethics committee of the Lagos University Teaching Hospital, and the Ethical committee of the Federal Neuropsychiatric Hospital, Yaba, Lagos before commencement.

Consenting and stable schizophrenic patients, who could understand English, and their non-patient significant relatives were serially recruited from the out-patient clinics of either hospital. The patients were assessed using the Structured Clinical Interview for DSM-IV disorders (SCID) - to verify the diagnosis of schizophrenia. The patient version of the Level of Expressed Emotion (LEE) Scale was administered. Participants also completed a socio demographic data questionnaire.9,10 The relatives were assessed using the 30item General Health Questionnaire $(\mathrm{GHQ})^{11}$, the relative version of the Level of Expressed Emotion Scale ${ }^{10}$ as well as a biographical data questionnaire.

The patients were predominantly young (mean age 39 years; $\mathrm{SD}=11.6)$, single (70.0\%), unemployed (68\%), and male (56.0\%), while the relatives were older ( 46.5 years; SD 14.1), predominantly female (54\%), married (52\%) and employed (62\%). The majority of subjects were of the Yoruba tribe. All the patients were on medication and clinically stable. The mean duration since their first episode was 5.62 years (SD 3.64) and 90\% reported spending less than 35 hours of weekly face-to-face contact with their significant relative. The significant relatives were mostly first degree relatives (68\%), and most often mothers. The mean GHQ score of the relatives was 1.8 (SD 2.8). The prevalence of 'high' expressed emotion was $46.0 \%$ and $50.0 \%$ for the patient and relative versions of the Level of Expressed Emotion Scale respectively. Criticism and emotional overinvolvement appeared to be stronger determinants and predictors of high expressed emotion. Relapse rates were higher among the high expressed emotion groups. Female gender and higher number of previous episodes were characteristics found to be associated with high expressed emotion. The suggestion is that these factors should be given more attention when designing intervention strategies. However it will be beneficial to study a larger population of subjects to make for more robust interpretations and conclusions.

RE Ogbolu1, JD Adeyemi1,2, AR Erinfolami1,2 1Department of Psychiatry, Lagos University Teaching Hospital, Idi-araba, Lagos, Nigeria

2 Department of Psychiatry, College of Medicine, University of Lagos, Idi-araba, Lagos, Nigeria 


\section{References}

1. Kuipers E. Expressed emotion in 1991. Social Psychiatry and Psychiatric Epidemiology 1992; 27: 1-3.

2. Butzlaff RL, Hooley JM. Expressed emotion and psychiatric relapse: $A$ meta-analysis. Archives of General Psychiatry 1998; 55: 547-552.

3. Brown GW, Carstairs GM, Topping G. Post-hospital adjustment of chronic mental patients. The Lancet 1958; 2:685-689.

4. Vaughn CE, Leff JP. The influence of family and social factors on the course of psychiatric illness. British Journal of Psychiatry 1976; 129: 125-137.

5. Cutting L, Docherty N. Schizophrenia outpatients' perceptions of their parents: Is expressed emotion a factor? Journal of Abnormal Psychology 2000; 109:266-272.

6. Reicher B, Adeyemi JD, Lawal RA, Famuyiwa OO, Haruna AY, Jibodu MO. Emotional reactions of relatives to schizophrenic patients in Lagos, Nigeria. African Journal of Medical Sciences 2003; 32(1):7-11.
7. Vaughn CE, Leff JP. The measurement of expressed emotions in the families of psychiatric patients. British Journal of Social and Clinical Psychology. 1976b; 15:157-165.

8. Jablensky A, Sartorius N, Ernberg G, et al. Schizophrenia manifestations, incidence and course in different cultures. A World Health Organization ten-country study. Psychology of Medicine Monograph Suppl. 1992; 20:1-97.

9. Basco MR, Bostic JQ, Davies D. Methods to improve diagnostic accuracy in a community mental health setting. American Journal of Psychiatry 2000; 157:1599-1605.

10. Cole JD, Kazarian SS. Predictive validity of the Level of Expressed Emotion (LEE) Scale; readmission follow-up data for 1, 2 and 5 year periods. Journal of Clinical Psychology 1993; 49(2):21 6-218.

11. Goldberg DP. General health questionnaire manual. Psychological Medicine 1997; 27:191-197.

\section{INSTRUCTIONS FOR AUTHORS}

\section{Instructions for Authors African Journal of Psychiatry (AJP)}

Material submitted for publication in the African Journal of Psychiatry (AJOP) is accepted on condition that it meets the requirement of the Editor-in-Chief The publisher reserves the copyright of the material published.

All authors must give consent to publication, and the AJOP does not hold itself responsible for statements made by contributors.

The Journal's primary aim is the publication of review and original articles Case reports, which require consent for publication of content, will be considered and if accepted, published as scientific letters. Scientific letters, which should be no more than 1000 words with a maximum of 10 references and 1 table or figure, will also include content such as pilot studies or those with smaller sample sizes. Letters to the editor are also published, and would generally be responses to published material. The Journal is aimed at specialist mental health care and other professionals working in the neurosciences as well as primary care practitioners. All material will be sent for peer review.

\section{Manuscript preparation}

1. Copies should be neatly typewritten, with double spacing and wide margins. The manuscript should be submitted electronically. Authors are required to state that their material is original and not previously published or currently submitted elsewhere

2. All abbreviations should be spelt out when first used in the text and thereafter used consistently.

3. Scientific measurements should be expressed in SI units throughout, with two exceptions: blood pressure should be given in $\mathrm{mmHg}$ and haemoglobin values in $\mathrm{g} / \mathrm{dl}$.

4. Author's full name \& surname, affiliation \& correspondence address (including email address) to be set out in full on title page of article.

5. All articles (review, original research ) are to have an $\mathbf{A b s t r a c t}$, giving a brief succinct overview of the article. The abstract should reflect the essence of the paper and be $\mathbf{2 0 0}$ to $\mathbf{2 5 0}$ words. For Original Research articles, the abstract should be structured as follows:- Objective, Method, Results and Conclusion Review articles should be approximately $\mathbf{5 0 0 0}$ words, and original articles approximately $\mathbf{3 0 0 0}$ words.

6. Authors must give a minimum of three key words, and should use the MeSH (Medical subject headings list of index medicus) cataloque.

7. A clear statement on ethical issues in clinical and animal research must be provided; conflict of interests and patient confidentiality issues must be indicated. This should appear in the Methods section and must contain the name of the Ethics Committee responsible as well as the name of the Chairperson or the person who approved the protocol. For case reports a statement needs to be included regarding consent to publish case material from the patient, failing which permission from the relevant ethics committee should be included as per the for clinical and animal research.

8. For multi authored papers, the International Committee of Medical Journal Editors (ICMIE) states that, there are three necessary conditions one must meet in order to claim (co) authorship:

1. Substantial contributions to conception and design, or acquisition of data, or analysis and interpretations of data.
2. Drafting the article or revising it critically for important intellectual content

3. Final approval of the version to be published.

Those, and only those who meet all three of the above stipulations, can be named authors, while those who meet only some of the requirements or otherwise facilitate the research by contributing to funding, data collection, editorial work etc. should be named in the 'Acknowledged' section.

Accordingly, multi-authored papers need a declaration of relative contribution.

\section{Illustrations}

1. Figures consist of all material which cannot be set in type, such as photographs and line drawings. Photographs should be forwarded electronically.

Tables and legends for illustrations should be typed on separate sheets and should be clearly identified. Tables should carry Roman numerals, thus I, II, III etc, and illustrations Arabic numerals, thus: 1, 2, 3, etc.

3. Where identification of a patient is possible from a photograph the author must submit a consent to publication signed by the patient, or by the parent or guardian in the case of a minor.

4. If any tables or illustrations submitted have been published elsewhere, written consent to republication should be obtained by the author from the copyright holder and the author(s)

\section{References}

1. References should be inserted at the end of the sentence, outside the full stop, as superior numbers, and should be listed at the end of the article in numerical order. Do not list them alphabetically.

. It is the author's responsibility to verify references from the original sources.

3. References should be set out in the Vancouver style, and only approved abbreviations of journal titles should be used; consult the List of Journals Indexed in Index Medicus for these details. Names and initials of all authors should be given unless there are more than six, in which case the six names should be given followed by "et al". First and last page numbers should be given.

Journal references should appear as follows:

a. Peter S, Jones A. Smith B. Acute hamstring injuries. Am J Sports Med 1994; 12(7):395-400.

Book references should be set out as follows:

a. Williams G. Textbook of Sports Medicine. 2nd Edition: Butterworth, 1989: 101-104

b. Vandermere P. Russel P. Biomechanics of the hip joint. In:Nordien PE. Jeffcoat A, eds, Clinical Biomechanics. Philadelphia:WB Saunders, 1990:472-479.

4. "Unpublished observations" and "personal communications" may be cited in the text, but not in the reference list.

Manuscripts accepted but not yet published can be included as references followed by "(in press)"

All manuscripts and correspondence should be emailed to Professor CP Szabo, email: christopher.szabo@wits.ac.za 